\title{
A population-based analysis of the risk of drug interaction between clarithromycin and statins for hospitalisation or death
}

\author{
Bita Mesgarpour ${ }^{1,2}$, Ghazaleh Gouya ${ }^{1}$, Harald Herkner ${ }^{3}$, Berthold Reichardt ${ }^{4}$ and Michael Wolzt ${ }^{\text {* }}$
}

\begin{abstract}
Background: Clarithromycin, known as a potent inhibitor of the cytochrome P450 isoenzyme CYP3A, may increase the plasma concentration of statins metabolized by this pathway; therefore, increase the risk of interaction with statins in reference to pharmacokinetic studies. This study aimed to characterize whether the concomitant use of a statin with clarithromycin is associated with serious outcomes among adult persons.

Methods: Health claims data of adult persons in the Regional Sickness Fund of Burgenland, Austria, who filled a prescription for clarithromycin between July 1, 2009 and June 30, 2012 were reviewed retrospectively. We assumed that the risk of hospitalisation increases acutely with the indication for taking an antibiotic, whereas statin use can be considered a chronic exposure with a low constant effect on hospitalisation. When defining the population as persons taking clarithromycin and the use of statins as the exposure we could achieve a comparable effect in both groups from the acute condition on hospitalisation. Therefore, we defined exposed patients as those who had overlapping treatment with a statin and unexposed controls as those who had filled a prescription for clarithromycin without concomitant statin therapy. Outcome was defined as a composite of hospital admission or death within 30 days after starting clarithromycin. We used generalised linear regression to model an association between outcome and exposure to statins.
\end{abstract}

Results: Among 28,484 prescriptions of clarithromycin, 2317 persons were co-exposed to statins. Co-administration of CYP3A4 metabolized statins and clarithromycin was associated with a 2.11 fold increased risk of death or hospitalisation (95\% confidence interval [Cl]: 1.79-2.48). This effect was explained by age, evidence of cardiovascular disease, diabetes mellitus and utilization of other antibiotics (multivariable adjusted risk ratio: 1.02, $95 \% \mathrm{Cl}$ : 0.85-1.22). The sensitivity analyses did not change the significance of effect.

Conclusions: The risk for hospitalisation or death in persons receiving clarithromycin increases with age and cardiovascular disease but is not causally associated with statin-clarithromycine co-administration.

Keywords: HMG-CoA reductase inhibitors, Drug interactions, Clarithromycin, Electronic health records, Cohort studies

\section{Background}

The potential of interaction between drugs that inhibit the CYP3A4 metabolic pathway and 3A4 substrates is well recognised [1-8]. However, the clinical relevance of this interaction is poorly defined and co-prescription of these medications is common in the clinical setting [9-12]. Macrolide antibiotics such as clarithromycin are known as potent inhibitors of CYP3A4, thus introducing the

\footnotetext{
* Correspondence: michael.wolzt@meduniwien.ac.at

1 Department of Clinical Pharmacology, Allgemeines Krankenhaus Wien, Medical University of Vienna, Währinger Gürtel 18-20, 1090 Vienna, Austria Full list of author information is available at the end of the article
}

potential for a pharmacological interaction with CYP3A4metabolised statins (atorvastatin, simvastatin and lovastatin). Concomitant use of macrolides with statins 3A4 substrate was detected from 1.6 to $6.3 \%$ in the electronic medical records databases $[9,11]$.

Studies showed that co-administration of clarithromycin have significant effect on pharmacokinetic parameters of simvastatin and atorvastatin, with an approximately tenfold or fourfold increase in drug exposure as measured by the area of the drug concentration versus time curve, respectively. It was also associated with approximately seven- to eightfold and greater than fivefold increase in 
maximum plasma concentration of simvastatin and atorvastatin, respectively [13, 14]. Study on the spontaneous adverse event reports, using the US Food and Drug Administration Adverse Event Reporting System database showed a six fold increase in the adverse event reporting rate and ratio (AERR) of rhabdomyolysis in concomitant use of simvastatin with CYP3A4 inhibitors compared to simvastatin without CYP3A4 inhibitors (56 events in $14,575,000$ prescriptions versus 62 events in 103,822,000 prescriptions) [15]. Although, several case reports support this interaction [16-23]. However, limited evidence from controlled studies confirms and measures the potential risk of serious adverse events due to this interaction $[9,24,25]$. Furthermore, none of them has the appropriate power to recognize a specific CYP3A4 inhibitor-statins interaction. In an effort to address the clinical impact of this drug-drug interaction, we did a cohort study to investigate the risk of hospitalization or death associated with co-prescription of a statin with clarithromycin.

\section{Results}

In total, 69,877 HMG CoA reductase inhibitors were prescribed in clarithromycin users (independent of coadministration) during the observation period (Fig. 1). A total of 28,484 prescriptions in 23,339 persons were included in the analyses. We determined 2317 coadministrations of statins and clarithromycin as exposed

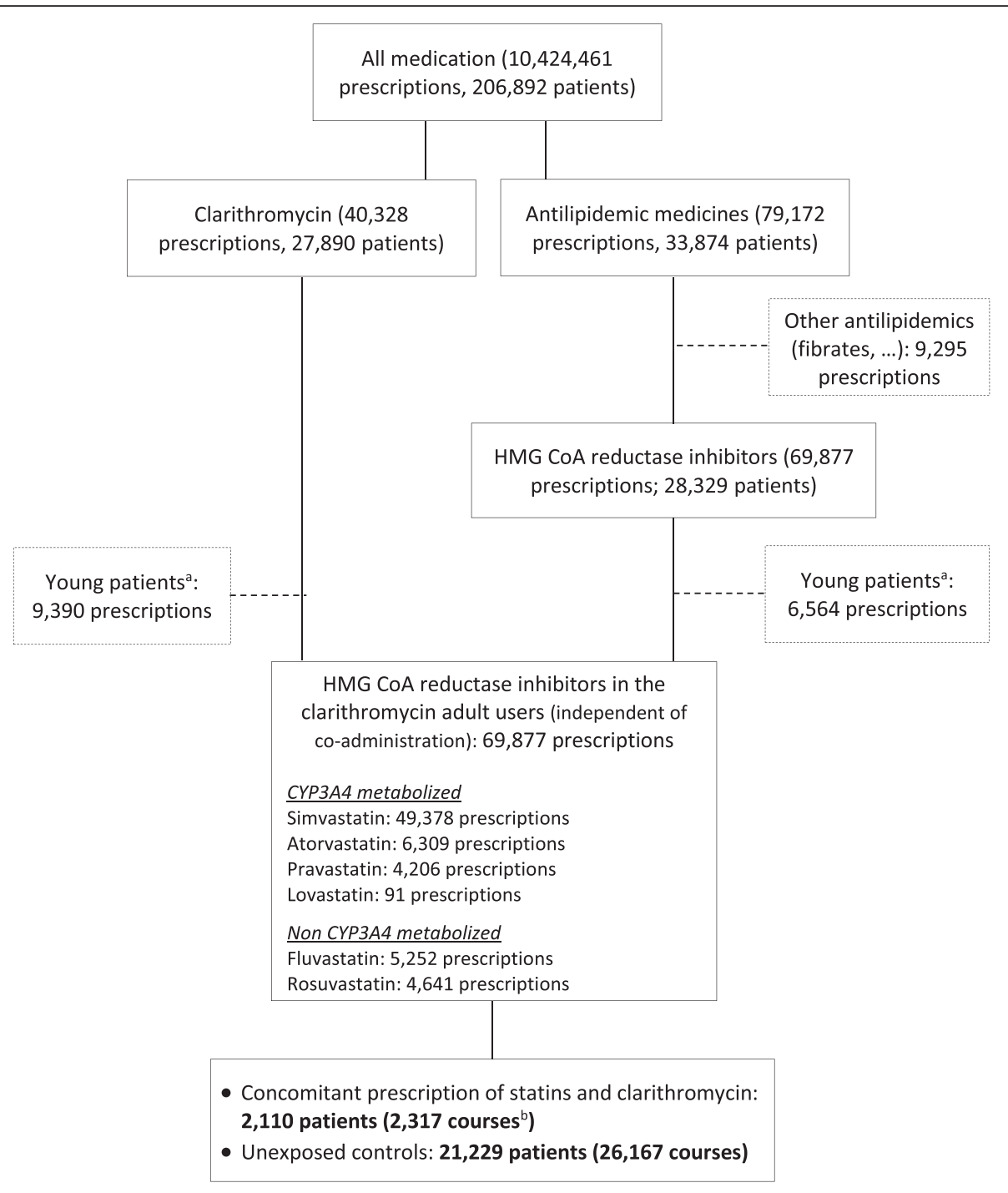

aless than 18 years old

${ }^{b}$ For persons who had multiple prescription of clarithromycin, we classified treatment as a new case if there was a gap of three months or more between the end date of utilization of clarithromycin and starting the new course.

Fig. 1 Clarithromycin and antilipidemic agents' utilization in BGKK from 2009.07.01 to 2012.06.30 
persons comparing to 26,167 prescriptions of clarithromycin only as unexposed control.

Person demographics and prescriptions are presented in Table 1. Among the persons who received co-administrations of statins and clarithromycin, 206 $(8.89 \%)$ hospitalizations or deaths were observed during the follow-up period. In the unexposed clarithromycin group, 1128 (4.31\%) persons were hospitalized or died during the observation period.

In the crude unadjusted analysis, current concomitant use of CYP3A4 metabolized statins with clarithromycin was associated with 2.11 (95 \% CI: 1.79-2.48) times higher risk for hospitalization or death compared to clarithromycin without current concomitant use of CYP3A4 metabolized statins (Table 2). Current age, treatment with other antibiotics, evidence of diabetes or cardiovascular disease each explained partly the effect of current statins on death or hospitalization in the bivariate analyses. In multivariate combination, however, these four variables fully account for the association between current statin use and the outcome of death or hospitalization (multivariable adjusted RR: 1.02 (0.85-1.22). The effects were virtually unchanged for persons younger or older than 65 years ( $p$-value for interaction 0.11$)$. There were also no other significant interactions on the effect of current statin use on the outcome.

We conducted a sensitivity analyses to explore the impact of frequency of clarithromycin use, hospital admission or death within 5 or 10 days and death or hospitalisation separately. Interaction of CYP3A4 metabolized statins and clarithromycin was not significantly different with restriction of exposed persons to those with first time prescription of clarithromycin ( $R R=0.96,95 \% \mathrm{CI}$ : $0.79-1.16)$ or on a one-time basis ( $R R=0.92,95 \%$ CI: 0.74-1.13). Results of the sensitivity analyses for death or hospitalization within 5 or 10 days $(\mathrm{RR}=0.78,95 \% \mathrm{CI}$ : $0.55-1.12$; $\mathrm{RR}=1.38,95 \% \mathrm{CI}: 0.95-2.00)$, only hospitalisation or only death within 30 days $(\mathrm{RR}=1.01,95 \% \mathrm{CI}$ : 0.84-1.21; $\mathrm{RR}=1.2,95 \%$ CI: 0.46-3.18) did not differ significantly from our predefined outcome, death or hospitalisation within 30 days.

\section{Discussion}

This population-based analysis of reimbursement data suggest that hospital admission and mortality are similar when clarithromycin is prescribed in the presence or absence of CYP3A4 substrate statins. This finding puts the pharmacokinetic drug interaction into clinical perspective.

Our study suggests a lack of a causal effect for current statin use on death or hospitalisation of clarithromycin users. Increased clinical risk is driven by the effects of age, evidence of diabetes or cardiovascular disease and treatment with other antibiotics.

Table 1 Patient demographics and prescriptions

\begin{tabular}{lll}
\hline & Exposed patients & Unexposed controls \\
\hline Age, mean \pm SD & $66.3 \pm 11.1$ & $46.8 \pm 17.2$ \\
Female & $1426(61.5 \%)$ & $16,990(64.9 \%)$
\end{tabular}

Concomitant statins

\begin{tabular}{|c|c|c|}
\hline \multicolumn{3}{|l|}{ CYP3A4 metabolized } \\
\hline Simvastatin & 1589 & - \\
\hline Atorvastatin & 195 & - \\
\hline Pravastatin & 151 & - \\
\hline Lovastatin & 2 & - \\
\hline \multicolumn{3}{|l|}{ Non CYP3A4 metabolized } \\
\hline Fluvastatin & 205 & - \\
\hline Rosuvastatin & 175 & - \\
\hline Current statin users & 1959 & - \\
\hline \multicolumn{3}{|l|}{ listory of disease $n(\%)^{a}$} \\
\hline Diabetes & $622(26.8 \%)$ & $1236(4.7 \%)$ \\
\hline Cardiovascular disease & $1906(82.3 \%)$ & 7704 (29.4 \%) \\
\hline Malignancy & $52(2.2 \%)$ & 337 (1.3\%) \\
\hline Autoimmune disorders & $27(1.2 \%)$ & 172 (0.7 \%) \\
\hline Antiplatelet drugs & $665(28.7 \%)$ & 860 (3.3 \%) \\
\hline P2Y12 inhibitors & $194(8.4 \%)$ & $136(0.5 \%)$ \\
\hline ASA \pm dipyridamole & $537(23.2 \%)$ & 757 (2.9\%) \\
\hline $\begin{array}{l}\text { Highly active antiretroviral } \\
\text { therapy }\end{array}$ & $0(0 \%)$ & $2(0 \%)$ \\
\hline \multicolumn{3}{|l|}{ Concomitant medications } \\
\hline Other antilipidemic agents & $116(5.0 \%)$ & $241(0.9 \%)$ \\
\hline Other systemic antibiotics & $684(29.5 \%)$ & $23,580(90.1 \%)$ \\
\hline
\end{tabular}

Drug-drug interaction

(synergistic effect) $^{b}$

Clarithromycin

Strong interaction

Moderate interaction

Weak interaction

Statins

Strong interaction

Moderate interaction

Weak interaction

$$
\begin{aligned}
& 78(3.4 \%) \\
& 882(38.1 \%) \\
& 581(25.1 \%)
\end{aligned}
$$

$312(1.2 \%)$

$2859(10.9 \%)$

$2768(10.6 \%)$

Drug-drug interaction

(antagonistic effect) $^{\mathrm{b}}$

\section{Clarithromycin}

Statins

Outcomes

$\begin{array}{lll}\text { No. of hospitalization } & 198(8.5 \%) & 1108(4.2 \%) \\ \text { No. of death } & 8(0.3 \%) & 20(0.1 \%)\end{array}$

ASA acetylsalicylic acid, CYP3A4 cytochrome P450 3A4, SD standard deviation ${ }^{\text {a } C o n c o m i t a n t ~ d i s e a s e ~ a s ~ s u g g e s t e d ~ b y ~ c o-m e d i c a t i o n ~}$

${ }^{\text {b }}$ See the details in supplementary methods 
Table 2 Risk estimate in current concomitant use of CYP3A4 metabolized statins with clarithromycin within 30 days

\begin{tabular}{llr}
\hline Risk of death or hospitalisation & RR $(95 \% \mathrm{Cl})$ & $P$ value \\
\hline Unadjusted risk & $2.11(1.79-2.48)$ & $<0.001$ \\
$\begin{array}{l}\text { Adjusted for } \\
\quad \text { Current age }{ }^{\mathrm{a}}\end{array}$ & $1.18(1.00-1.40)$ & 0.049 \\
$\quad$ Diabetes mellitus & $1.60(1.34-1.91)$ & $<0.001$ \\
$\quad$ Cardiovascular disease & $1.33(1.12-1.57)$ & 0.001 \\
$\quad$ Other antibiotics & $1.55(1.28-1.87)$ & $<0.001$ \\
$\begin{array}{l}\text { Multivariable adjusted for } \\
\quad \begin{array}{l}\text { age, diabetes mellitus, } \\
\text { cardiovascular disease }\end{array}\end{array}$ & $1.02(0.85-1.22)$ & 0.85 \\
$\quad$ and other antibiotic therapy & & \\
\hline
\end{tabular}

Estimates come from a generalised linear model using a log link function; confidence intervals are based on robust standard errors

${ }^{a}$ current age as indicator variable of quintiles

Our findings are consistent with a population-based cohort study on the UK Health Improvement Network (THIN) database, which detected no difference in the relative hazard of muscle toxicity, renal dysfunction or hepatic dysfunction in patients prescribed a statin CYP3A4 substrate versus a statin non-CYP3A4 substrate in co-medication with another CYP3A4 inhibitor [26]. Our present study extends these findings, since it has focussed on a specific CYP3A4 inhibitor in a large population. Further, we adjusted our data for all possible drug interactions of clarithromycin and statins such as antiretroviral therapy since our data was not limited to general physicians' prescriptions. The number of prescriptions of some classes of drugs, e.g. antiretroviral treatments, was however too small to draw meaningful conclusions and to rule out relevant clinical effects. We also adjusted for prescription of other systemic antibiotics during the observation period as proxy for the severity of disease.

Likewise, a Canadian population-based, nested casecontrol study has evaluated drug-drug interaction of donepezil, which is metabolized by CYP3A4, and clarithromycin. The risk of adverse cardiovascular events was not increased by concurrent administration of clarithromycin in elderly donepezil users compared with azithromycin, which does not interact with the cytochrome P-450 system $(\mathrm{OR}=0.67 ; 95 \% \mathrm{CI}: 0.28-1.63 ; p=0.38)$ [27]. In contrast, a recent Canadian population-based cohort study detected a higher risk for hospitalization with rhabdomyolysis ( $\mathrm{RR}=2.17,95 \% \mathrm{CI}: 1.04-4.53)$, acute kidney injury $(\mathrm{RR}=1.78,95 \% \mathrm{CI}, 1.49-2.14)$ and for allcause mortality ( $R R=1.56,95 \% \mathrm{CI}: 1.36-1.80)$ in older people associated with co-prescription of a CYP3A4 metabolized statin with clarithromycin or erythromycin compared with azithromycin. [25] Another Canadian population-based cohort study found that co-prescription of clarithromycin versus azithromycin with a calcium- channel blocker was associated with a higher risk of hospitalization with acute kidney injury ( $\mathrm{OR}=1.98,95 \%$ CI: 1.68-2.34) [28].

The result of our study is at variance with a study on dispensed prescriptions of lipid-lowering drugs using administrative claims data from diverse regions in the United States. The increased risk of hospitalization due to myopathy, renal and hepatic adverse medical events has been identified in lipid-lowering drug users with coadministration of CYP3A4 inhibitors $(\mathrm{RR}=6.01,95 \% \mathrm{CI}$ : 2.08-17.38; $\mathrm{RR}=2.29,95 \% \mathrm{CI}: 1.62-3.23$; and $\mathrm{RR}=2.55$, $95 \%$ CI: 1.76-3.70, respectively) [24]. Although, this study has the limitation of a case-control study design, not controlling of confounding variables like history of other disease, as well as lack of subgroup analysis for detecting the effect of statins-CYP3A4 inhibitors interaction. Moreover, monotherapy with statins are generally well tolerated and present a safe profile. Therefore, the choice of analysis, comparing the co-administration of statins and clarithromycin with monotherapy of statins or monotherapy of clarithromycin might affect the risk of serious adverse events of co-administration.

As we conducted a database analysis of prescription data, medication adherence is not accounted for. In addition, the possibility of a transient interruption of statin intake during clarithromycin therapy by physician advice was not accessible from our database. These factors would have concealed a drug-drug interaction.

This study does not indicate an increased risk for hospitalisations or death when clarithromycin is prescribed with statins in a cohort of unselected persons in primary care. The well-described pharmacokinetic interaction of these medicines does not result in an increased precipitation of serious clinical endpoints.

\section{Methods \\ Population}

We established a cohort of persons aged 18 years or older in the Regional Sickness Fund of Burgenland (BGKK) who filled a prescription for reimbursement of clarithromycin between July 1, 2009 and June 30, 2012. The Austrian insurance system provides almost complete coverage of health care for all residents. According to their current employment or providence of residence, the membership to the regional Burgenland Sickness Fund is assigned. We received data from outpatient as well as inpatient medical services covered by the health insurance fund, which were stored in the respective anonymised dataset, including demographic data, information on hospital admissions and discharges with primary diagnoses coded using the International Classification of Diseases (ICD) system and drug prescriptions received. The data linkage with vital status of persons was provided by Statistik Austria. There was no ICD information of the extramural medical care 
included. The date of dispensing clarithromycin served as the date of cohort entry (entry date). For persons who had multiple prescription of clarithromycin, we classified treatment as a new case if there was a gap of 3 months or more between the end date of utilization of clarithromycin and starting the new course. The initial unit of analysis was a course of clarithromycin treatment (accounting for withinperson correlation). For a secondary analysis, we handled single persons as the unit of analysis and used the first course of clarithromycin, ignoring further courses of clarithromycin treatment.

For each person, we determined the total duration of clarithromycin treatment using Defined Daily Dose (DDD) information from WHO; this information was used as a covariate in the analyses.

\section{Exposed persons}

We assumed that the risk of hospitalisation increases acutely with the indication for taking an antibiotic, whereas statin use can be considered a chronic exposure with a low constant effect on hospitalisation. When defining the population as persons taking clarithromycin and the use of statins as the exposure we could achieve a comparable effect in both groups from the acute condition on hospitalisation. Given that we were able to adjust for confounding by indication for statin use we assume that this approach enabled us to appropriately examine the drug-drug interaction of statins and clarithromycin. Given the absence of detailed clinical information in the database, we were not able to adjust for factors related to the indication of clarithromycin use, therefore an approach of comparing clarithromycin exposure in a population of statin users appeared less promising. Therefore, we identified persons who had a statin co-prescribed during clarithromycin treatment. We defined exposed persons as those who were treated with clarithromycin and had overlapping treatment with a statin at least for 1 day (concomitant users). We defined 'current statin users' if a person had utilized a statin within 5 days before the entry date. Exposure status was stratified by statins metabolized by the enzyme CYP3A4 (atorvastatin, lovastatin, pravastatin, simvastatin) and those metabolized by the CYP2C9 (fluvastatin, rosuvastatin).

\section{Unexposed persons}

Persons that had no overlapping statin exposure during their clarithromycin treatment were determined as controls.

\section{Outcome}

Outcome was defined as a composite of hospital admission or death within 30 days. In separate sensitivity analyses, we assigned hospital admission as endpoint if it happened within 5, 10 and 30 days after starting clarithromycin (entry date).

\section{Observation time}

We defined the exit date as the date of the occurrence of the outcome or the end of the observation time. $\mathrm{Ob}$ servation time $=$ exit date - entry date.

\section{Covariates}

Prescriptions of drugs used to treat diabetes (ATC code A10), cardiovascular disease (ATC code B01, C01, C02, C03, C04, C07, C08, C09), malignancy (ATC code: L01, L02B, L03), autoimmune disorders (ATC code: L04) and other antilipidemic (ATC code: C10AB, C10AC, C10AD, $\mathrm{C} 10 \mathrm{AX}$ and $\mathrm{C} 10 \mathrm{~B})$ before the entry date used to identify relevant disease in cases and controls. We identified antiplatelet drugs including P2Y12 inhibitors (ATC code: $\mathrm{B} 01 \mathrm{AC} 04, \mathrm{~B} 01 \mathrm{AC} 22, \mathrm{~B} 01 \mathrm{AC} 24)$, aspirin and combination of aspirin and dipyridamole (ATC code: B01AC06, B01AC30) as proxies for manifestation of cardiovascular disease. Highly active antiretroviral therapy (HAART) used to treat HIV infection has been detected based on recommended guidelines [29, 30]. We defined the person's history of the above disease if the relevant medications have been prescribed at least 6 month before the cohort entry.

Clarithromycin is metabolized by the enzyme CYP3A4 (CYP3A4 substrate) and acts as an inhibitor of the metabolizing enzyme CYP3A4. Therefore, we defined the list of agents inhibiting and inducing CYP3A metabolism as well as other drugs with synergistic/antagonistic effects on clarithromycin or statins to detect co-administration of each one (as confounder of the effect of clarithromycin on statins) during the observation period (Additional file 1). We used prescription of other systemic antibiotics (ATC code-J01) during the observation period as proxy for the severity of disease. We compared the frequency of the outcome between exposed persons and unexposed controls. Baseline data, demographics and risk or exposure related covariates were tabulated and compared using two-sided hypothesis tests.

\section{Statistical analyses}

We used a generalised linear regression model to assess the association between the outcome and the exposure to statins with a log-link function to get directly risk ratios (RR) as the measure of effect. For the main analysis, the exposure was current concomitant use of CYP3A4 metabolised statins and the outcome was death or hospitalisation within 30 days. The unit of analysis for these models was a course of clarithromycin treatment. We allowed for within person correlation in case of repeated treatment courses by using robust standard errors. To adjust for potential confounding, we also included candidate covariates if they changed the main effect by $>10 \%$ in a bivariate analysis and if they were not considered moderator variables. We tested for first order interactions by including interaction terms into the models. We performed several 
sensitivity analyses to assess the robustness of our estimates. Accordingly, we repeated our analyses using several definitions of exposure status by exposure time and statin type. We also analysed hospitalisation and mortality separately. Likewise, the analyses have been repeated by allowing for varying the outcome time definitions. We also used several modelling approaches (including logistic regression and proportional hazards regression) and methods for handling correlated data (robust standard errors or random effects models).

Data analysis was performed using Microsoft Excel and Stata 11 (Stata Corp, College Station, TX). A two-sided $p$ value $<0.05$ was considered statistically significant.

\section{Additional file}

\section{Additional file 1: Drug Interactions for clarithromycin and statins.}

Synergistic Interactions with Clarithromycin. Major Inducers of CYP3A4. Synergistic Interactions with Simvastatin, Lovastatin, Pravastatin, Fluvastatin, Atorvastatin, Rosuvastatin. Antagonistic Interaction with Simvastatin, Lovastatin, Pravastatin, Fluvastatin, Atorvastatin. (PDF 470 kb)

\section{Abbreviation}

AERR: Adverse event reporting rate and ratio; BGKK: Regional Sickness Fund of Burgenland; DDD: Defined Daily Dose; ICD: International Classification of Diseases; THIN: The UK Health Improvement Network.

\section{Competing interests}

This study was supported in part by a grant from AstraZeneca, Austria. The funder had no role in study design, data collection and analysis, decision to publish or preparation of the manuscript. The authors alone are responsible for the content and writing of the paper.

\section{Authors' contributions}

Conception of research idea: BM, GG, HH, MW; Study Design: BM, HH; Data collection: BR, GG; Data management: BM, BR; Data analysis: BM, HH; Drafting paper: $\mathrm{BM}, \mathrm{HH}, \mathrm{MW}$. All authors read and approved the final manuscript.

\section{Author details}

'Department of Clinical Pharmacology, Allgemeines Krankenhaus Wien, Medical University of Vienna, Währinger Gürtel 18-20, 1090 Vienna, Austria. ${ }^{2}$ Digestive Diseases Research Institute (DDRI), Tehran University of Medical Sciences (TUMS), Tehran, Iran. ${ }^{3}$ Department of Emergency Medicine, Medical University of Vienna, Vienna, Austria. ${ }^{4}$ Sickness Fund Burgenland, Eisenstadt, Austria.

Received: 3 July 2015 Accepted: 13 October 2015

Published online: 24 October 2015

\section{References}

1. Cheng JW, Frishman WH, Aronow WS. Updates on cytochrome p450-mediated cardiovascular drug interactions. Dis Mon. 2010;56:163-79.

2. Jana S, Paliwal J. Molecular mechanisms of cytochrome p450 induction: potential for drug-drug interactions. Curr Protein Pept Sci. 2007;8:619-28.

3. Kalra BS. Cytochrome P450 enzyme isoforms and their therapeutic implications: an update. Indian J Med Sci. 2007;61:102-16.

4. Liu YT, Hao HP, Liu CX, Wang GJ, Xie HG. Drugs as CYP3A probes, inducers, and inhibitors. Drug Metab Rev. 2007:39:699-721.

5. Pelkonen O, Turpeinen M, Hakkola J, Honkakoski P, Hukkanen J, Raunio H. Inhibition and induction of human cytochrome P450 enzymes: current status. Arch Toxicol. 2008:82:667-715.

6. Tanaka E. Clinically important pharmacokinetic drug-drug interactions: role of cytochrome P450 enzymes. J Clin Pharm Ther. 1998;23:403-16.

7. Zhou SF. Drugs behave as substrates, inhibitors and inducers of human cytochrome P450 3A4. Curr Drug Metab. 2008;9:310-22.
8. Zhou SF, Xue CC, Yu XQ, Li C, Wang G. Clinically important drug interactions potentially involving mechanism-based inhibition of cytochrome P450 3A4 and the role of therapeutic drug monitoring. Ther Drug Monit. 2007;29:687-710.

9. Bakhai A, Rigney U, Hollis S, Emmas C. Co-administration of statins with cytochrome P450 3A4 inhibitors in a UK primary care population. Pharmacoepidemiol Drug Saf. 2012;21:485-93.

10. Devold HM, Molden E, Skurtveit S, Furu K. Co-medication of statins and CYP3A4 inhibitors before and after introduction of new reimbursement policy. Br J Clin Pharmacol. 2009;67:234-41.

11. Ming EE, Davidson MH, Gandhi SK, Marotti M, Miles CG, Ke X, et al. Concomitant use of statins and CYP3A4 inhibitors in administrative claims and electronic medical records databases. J Clin Lipidol. 2008;2:453-63.

12. Ratz Bravo AE, Tchambaz L, Krahenbuhl-Melcher A, Hess L, Schlienger RG, Krahenbuhl $S$. Prevalence of potentially severe drug-drug interactions in ambulatory patients with dyslipidaemia receiving HMG-CoA reductase inhibitor therapy. Drug Saf. 2005;28:263-75.

13. Amsden GW, Kuye O, Wei GC. A study of the interaction potential of azithromycin and clarithromycin with atorvastatin in healthy volunteers. J Clin Pharmacol. 2002:42:444-9.

14. Jacobson TA. Comparative pharmacokinetic interaction profiles of pravastatin, simvastatin, and atorvastatin when coadministered with cytochrome P450 inhibitors. Am J Cardiol. 2004;94:1140-6.

15. Rowan C, Brinker AD, Nourjah P, Chang J, Mosholder A, Barrett JS, et al. Rhabdomyolysis reports show interaction between simvastatin and CYP3A4 inhibitors. Pharmacoepidemiol Drug Saf. 2009;18:301-9.

16. Arnold C, Lamy O, Hagmann N. [88 years old woman with acute muscular weakness and diffuse muscular pain: have you thought about the drugs?]. Praxis (Bern 1994). 2010;99(24):1507-11.

17. Grunden JW, Fisher KA. Lovastatin-induced rhabdomyolysis possibly associated with clarithromycin and azithromycin. Ann Pharmacother. 1997;31:859-63.

18. Kahri AJ, Valkonen MM, Vuoristo MK, Pentikainen PJ. Rhabdomyolysis associated with concomitant use of simvastatin and clarithromycin. Ann Pharmacother. 2004:38:719.

19. Lee AJ, Maddix DS. Rhabdomyolysis secondary to a drug interaction between simvastatin and clarithromycin. Ann Pharmacother. 2001:35:26-31.

20. Molden E, Andersson KS. Simvastatin-associated rhabdomyolysis after coadministration of macrolide antibiotics in two patients. Pharmacotherapy. 2007;27:603-7

21. Page SR, Yee KC. Rhabdomyolysis in association with simvastatin and dosage increment in clarithromycin. Intern Med J. 2014;44:690-3.

22. Trieu J, Emmett L, Perera C, Thanakrishnan K, Van Der Wall H. Rhabdomyolysis resulting from interaction of simvastatin and clarithromycin demonstrated by Tc-99 m MDP scintigraphy. Clin Nucl Med. 2004;29:803-4.

23. Wagner J, Suessmair C, Pfister HW. Rhabdomyolysis caused by comedication with simvastatin and clarithromycin. J Neurol. 2009;256:1182-3.

24. Cziraky MJ, Willey VJ, McKenney JM, Kamat SA, Fisher MD, Guyton JR, et al. Statin safety: an assessment using an administrative claims database. Am J Cardiol. 2006;97:61C-8.

25. Patel AM, Shariff S, Bailey DG, Juurlink DN, Gandhi S, Mamdani M, et al Statin toxicity from macrolide antibiotic coprescription: a population-based cohort study. Ann Intern Med. 2013;158:869-76.

26. Rowan CG, Brunelli SM, Munson J, Flory J, Reese PP, Hennessy S, et al. Clinical importance of the drug interaction between statins and CYP3A4 inhibitors: a retrospective cohort study in The Health Improvement Network. Pharmacoepidemiol Drug Saf. 2012;21:494-506.

27. Hutson JR, Fischer HD, Wang X, Gruneir A, Daneman N, Gill SS, et al. Use of clarithromycin and adverse cardiovascular events among older patients receiving donepezil: a population-based, nested case-control study. Drugs Aging. 2012;29:205-11.

28. Gandhi S, Fleet JL, Bailey DG, McArthur E, Wald R, Rehman F, et al. Calcium-channel blocker-clarithromycin drug interactions and acute kidney injury. JAMA. 2013;310:2544-53.

29. World Health Organization. Antiretroviral therapy for HIV infection in adults and adolescents. Geneva: World Health Organization (WHO); 2010.

30. Panel on Antiretroviral Guidelines for Adults and Adolescents. Guidelines for the Use of Antiretroviral Agents in HIV-1-Infected Adults and Adolescents. Department of Health and Human Services. Available at http://aidsinfo.nih.gov/ contentfiles/lvguidelines/AdultandAdolescentGL.pdf. Accessed in Jan. 2015. 